\section{Australian Journal of \\ Crop Science}

\title{
Development of clonal seedlings of promising Conilon coffee (Coffea canephora) genotypes
}

\author{
André Monzoli Covre ${ }^{1 *}$, Luciano Canal ${ }^{1}$, Fábio Luiz Partelli ${ }^{1}$, Rodrigo Sobreira Alexandre ${ }^{2}$, Adésio \\ Ferreira $^{3}$, Henrique Duarte Vieira ${ }^{4}$
}

${ }^{1}$ Universidade Federal do Espírito Santo, Departamento de Ciências Agrárias e Biológicas, Rodovia BR-101

Norte, Km 60, Bairro Litorâneo, CEP 29932-540, São Mateus, ES, Brazil

${ }^{2}$ Universidade Federal do Espírito Santo, Departamento de Ciências Florestais e da Madeira, Avenida

Governador Lindemberg, 316, Centro, CEP 29550-000, Jerônimo Monteiro, ES, Brazil

${ }^{3}$ Universidade Federal do Espírito Santo, Departamento de Produção Vegetal, Alto Universitário, $\mathrm{s} / \mathbf{n}^{\circ}$,

Guararema, Cx. postal 16, CEP 29500-000, Alegre, ES, Brazil

${ }^{4}$ Universidade Estadual do Norte Fluminense Darcy Ribeiro Centro de Ciências e Tecnologias Agrárias, Avenida Alberto Lamego, 2.000, Parque Califórnia, CEP 28013-602, Campos dos Goytacazes, RJ, Brazil

\section{*Corresponding author: andre-covre@hotmail.com}

\begin{abstract}
Conilon coffee is a highly heterozygous diploid plant that is also allogamous, producing seeds through cross-fertilisation. Due to their allogamy, plants of seminiferous origin are highly heterogeneous, exhibiting high morphological and genetic diversity. However, propagation through cutting guarantees the maximum homogeneity of fields, particularly in grain maturation, among other desirable characteristics. Currently, the majority of commercial Conilon coffee is planted using seedlings propagated by cuttings. Studying the juvenile behaviour of different clonal genotypes of the Conilon coffee plant represents an important tool to assist with the establishment of this crop. The objective of this study was to assess the growth, development and genetic diversity in several promising genotypes of the Conilon coffee plant based on the morphological characteristics of seedlings propagated by cutting. We used seedlings Conilon coffee of 33 genotypes selected by coffee growers, and with great productive potential, and genotype 02 of the Emcapa 8111 variety. Based on morphological characteristics linked to the growth and development of the aboveground and root systems of the genotypes, their phenotypic correlations were estimated, along with the genetic diversity among genotypes, using Tocher's optimisation method and the unweighted peer group method with arithmetic mean. Genotypes A1, P2, Z39, Ouro Negro, Tardio C, Cheique, and Z29 exhibited a higher Dickson quality index (between 0.40 and 0.56 ). Genetic variability exists among the seedlings of the 34 genotypes of Conilon coffee studied. The most dissimilar genotypes were CHR, 18, and Z39, which remained in isolated groups in all experiments.
\end{abstract}

Keywords: Coffea canephora; Genetic diversity; Variability; Vegetative propagation; Phenotypic correlation.

Abbreviations: ADM_aboveground dry matter; ANA_National Water Agency; CONAB_National Food Supply Agency; DAPC_days after planting by cutting DQI_Dickson quality index; EMS_etiolation of the main stem; ICO_International Coffee Organization; LA_leaf area; LDM_leaf dry matter; LLR_length of largest root; MAPA_Brazilian Ministry of Agriculture; NIPS_number of internodes on the primary stem; NISS_number of internodes on the secondary stem; NOS_number of orthotropic shoots; NPR_number of primary roots; NPTB_number of plagiotropic branches; PSBD_primary stem base diameter; PSH_primary stem height; RDM_root dry matter; RV_root volume; SDM_stem dry matter; SLM_specific leaf mass; SSBD_secondary stem base diameter; SSH_secondary stem height; TDM_total dry matter; and UPGMA_hierarchical unweighted pair group method with arithmetic mean.

\section{Introduction}

The genus Coffea encompasses at least 124 species, with Coffea arabica L. and C. canephora Pierre ex A. Froehner having commercial significance (Davis et al., 2011). Global coffee production in 2014 surpassed 141.85 million bags, of which $40.3 \%$ were $C$. canephora, produced mainly in developing countries (ICO, 2015). In the 2014 harvest, the Brazilian production of $C$. arabica (Arabica coffee) and $C$. canephora (Conilon and/or Robusta coffee) was 32.3 and 13.0 million bags, respectively, in an area of 1.95 million hectares (CONAB, 2015). In Brazil, the main conilon coffee producing states are Espírito Santo, Rondônia and Bahia, which together account for $95.6 \%$ of national production
(CONAB, 2015). Conilon coffee is a highly heterozygous (2n $=2 \mathrm{x}=22$ chromosomes) diploid plant that is also allogamous, producing seeds through cross-fertilisation (Denoeud et al., 2014). Due to their allogamy, plants of seminiferous origin are highly heterogeneous, exhibiting high morphological and genetic diversity (Conagin and Mendes, 1961; Bragança et al., 2001). However, propagation through cutting has been used for many years because it has several advantages, such as the retention of genetic characteristics of the stock plant, greater crop homogeneity, increased productivity, and the ability to separate crops based on their maturation cycle (Bragança et al., 2001; Partelli et al., 
2014b). Genetic variability in the population is required for any genetic improvement program because it allows the selection of superior genotypes (Ivoglo et al., 2008) that are adapted to the soil and climate conditions of the cultivation region. Several authors working with different genotypes of C. canephora, have obtained success in the identification of genotypes adapted to new growing regions (Barbosa et al., 2014), of materials more dissimilar for the establishment of programs of directed crosses (Cecon et al., 2008) and in population base training for later selection and production of hybrid (Ivoglo et al., 2008). Denoeud et al. (2014) found $C$. canephora to be a useful reference species for understanding the evolution of genomic structure in asterid angiosperms. Genotypes of Conilon coffee exhibit countless differences, including polyclonal varieties (Fonseca et al., 2004), a group of clones that are generally grouped by maturation cycle: early, medium, late and very late (Partelli et al., 2014b). A physiologically viable cutting method has been developed, guaranteeing the maximum homogeneity of fields, particularly in grain maturation, among other desirable characteristics (Weigel and Jurgens, 2002; Paiva et al., 2012; Partelli et al., 2014a). Currently, the majority of commercial Conilon coffee is planted using seedlings propagated by cuttings. Knowing the characteristics of seedlings in each genotype becomes an important tool in planting coffee fields (Covre et al., 2013). Thus, the objective of the present study was to assess the growth, development and genetic divergence of several promising genotypes of Conilon coffee based on the morphological characteristics of seedlings propagated by cutting.

\section{Results and Discussion}

\section{Growth and development initial}

The genotypes were divided into two groups based on leaf area (Table 1). The group composed of genotypes Z39, Z29, Z40, Bamburral, Tardio C, Z37, A1, Z18, 18, Cheique, P2, Z36, Ouro Negro, Pirata, Peneirão, 700, Z38, Z21 and 02 Emcapa represented the genotypes with the greatest LA. Greater LA implies greater surface to capture light, resulting in higher photosynthetic rates and leading to greater initial plant growth (Partelli et al., 2006).

Significant differences in NOS were not observed among genotypes, demonstrating that this is a characteristic of the species that does not vary with genotype within the species (Table 1). Low NOS observed may be associated with small seedling age. However, after transplanting of seedlings conilon coffee techniques are applied to induce sprouting of orthotropic branches, such as bending, which provides standardization in the number of orthotropic branches per plant (Schmidt et al., 2014). In adults coffee productivity is directly related to the amount of NOS and plagiotropic branches, since it increases the production area (Tomaz et al., 2005).

The genotypes could also be placed in two groups based on variable NPTB, with greater values in the genotypes Z39, Z36, Tardio C, A1, Z38, Bamburral, Z21, Peneirão, Cheique, L80, Precoce V and Bicudo. The PSH characteristic was observed the formation of three distinct groups among genotypes. Second Bonomo et al. (2004), height plant is an important component in the productivity of adult coffee, since it indicates a higher number of productive nodes, therefore, higher number of plagiotropic branches. For the variables PSBD and NIPS, there was greater variation in the genotypes, classifying them into four groups. The group with the highest values comprised the genotypes P2, Z39, A1, Peneirão, Cheique, Bamburral, Bicudo, Tardio C, Z37, Z21,
Z38, Z29, Ouro Negro and L80 for PSBD; genotype 18 had the highest value for NIPS (Table 1). Higher values for NPTB and PSBD are desirable, as they exhibit high correlation with productivity in future harvests (Bustamante et al., 2004; Contarato et al., 2010), and productivity is the main objective and selection criteria in coffee cultivation (Oliveira et al., 2011; Rodrigues et al., 2014). No significant differences were observed among the analysed genotypes in SSH, SSBD, or NISS (Table 2). Two groups emerged for the variables PSE, LLR, NPR, and RV. Genotypes P2, A1, Z39, and Cheique exhibited a greater RV compared with the others. According to Contarato et al. (2010), plants with well-developed root systems are capable of exploring a greater volume of soil, achieving greater efficiency in absorption of water and nutrients. DaMatta et al. (2007) and Partelli et al. (2014a) report that the growth of the root system in Conilon coffee varies with the propagation method and genotype studied, with great differences in diverse characteristics, such as length, distribution, architecture, and depth of the root system.

During seedling development, it is important to assess not only total dry matter accumulation but also its allocation in different parts of the seedling (Paiva et al., 2009; Covre et al., 2013). Genotype Z39 showed increased development relative to the accumulation of dry matter in all parts of the seedling, indicating appropriate balance between the shoot and root of the plants, whereas lower biomass production performance was observed in genotypes L80, AT Bahia, Graudão, CHR, and B01, which exhibited lower values of all variables analysed (Table 3). Plant biomass production is an important and highly consistent characteristic in the assessment of plant species development, complementing height data (Paiva et al., 2009; Covre et al., 2013). The LDM data suggested the formation of three groups, with values between 1.81 (A1) and 2.49 (Z39) $\mathrm{mg} /$ plant (Table 3), partially corroborating the results of Covre et al. (2013). For the variables SLM, SDM, RDM, and TDM, the genotypes could be placed in two groups. For ADM, the genotypes Z39, P2, Z29, Z40, Bamburral, A1, and Tardio C composed the group with the highest values (Table 3). For the $\mathrm{ADM} / \mathrm{RDM}$ ratio, the genotypes were classified into three groups, the best performing group presented values between $3.50(\mathrm{Z} 38)$ and 4.63 (Z40) (Table 3). A higher ADM/RDM ratio suggests that these genotypes possess more vigorous development aboveground compared with belowground. According to Contarato et al. (2010), the equilibrium between aboveground and root development is intimately related to the development and productivity of coffee plants, such that ideal genotypes exhibit high aboveground biomass production, followed by a highly developed root system. Based on the DQI assessment, the best performing group presented values between 0.40 (Z29) and 0.56 (A1) (Table 3). Covre et al. (2013), assessing Conilon coffee seedlings of the 13 genotypes of the clonal variety Vitória Incaper 8142, observed DQI values between 0.35 (V4) and 0.45 (V10). Dardengo et al. (2013), assessing the growth and quality of Conilon coffee seedlings (variety Robusta Tropical) produced in different containers and levels of shade, observed DQI values between 0.35 and 0.54 for pots and between 0.35 and 0.58 for seedlings grown in bags. Andrade Júnior et al. (2013), assessing Conilon coffee seedlings propagated by grafting and cuttings, found values of 0.63 for vegetative propagation and 1.32 for grafting, using seedlings $C$. canephora of seminiferous origin of the Robusta Tropical variety, as rootstock, as graft six clones of Vitória variety. The Dickson index is considered a good indicator of seedling quality as it accounts for the robustness and equilibrium of biomass distribution (Covre et al., 2013). According to Dickson 
Table 1. Morphological characteristics of seedlings of different Conilon coffee genotypes.

\begin{tabular}{|c|c|c|c|c|c|c|c|}
\hline Genotypes & $\mathrm{LA}^{1}$ & $\mathrm{NOS}^{2}$ & NPTB $^{3}$ & $\mathrm{PSH}^{4}$ & $\mathrm{PSBD}^{5}$ & PSH/PSBD ${ }^{6}$ & $\mathrm{NIPS}^{7}$ \\
\hline Tardio C & $532.01 \mathrm{a}^{*}$ & $1.8 \mathrm{a}$ & $2.0 \mathrm{a}$ & $10.40 \mathrm{c}$ & $3.83 \mathrm{a}$ & $2.75 \mathrm{~b}$ & $3.6 \mathrm{~b}$ \\
\hline 18 & $488.33 \mathrm{a}$ & $1.0 \mathrm{a}$ & $0.6 \mathrm{~b}$ & $15.44 \mathrm{a}$ & $3.40 \mathrm{~b}$ & $4.52 \mathrm{a}$ & $5.0 \mathrm{a}$ \\
\hline Ouro Negro & $470.54 \mathrm{a}$ & $1.2 \mathrm{a}$ & $1.0 \mathrm{~b}$ & $13.12 \mathrm{~b}$ & $3.74 \mathrm{a}$ & $3.58 \mathrm{~b}$ & $4.2 \mathrm{~b}$ \\
\hline $\mathrm{Z} 36$ & $476.34 \mathrm{a}$ & $1.2 \mathrm{a}$ & $2.2 \mathrm{a}$ & $9.68 \mathrm{c}$ & $3.46 \mathrm{~b}$ & $2.78 \mathrm{~b}$ & $3.0 \mathrm{c}$ \\
\hline Z21 & $425.39 \mathrm{a}$ & $1.2 \mathrm{a}$ & $1.4 \mathrm{a}$ & $16.10 \mathrm{a}$ & $3.78 \mathrm{a}$ & $4.33 \mathrm{a}$ & $4.0 \mathrm{~b}$ \\
\hline Z37 & $495.03 \mathrm{a}$ & $1.6 \mathrm{a}$ & $0.4 \mathrm{~b}$ & $17.38 \mathrm{a}$ & $3.82 \mathrm{a}$ & $4.55 \mathrm{a}$ & $4.0 \mathrm{~b}$ \\
\hline Z18 & $489.76 \mathrm{a}$ & $1.4 \mathrm{a}$ & $0.8 \mathrm{~b}$ & $15.58 \mathrm{a}$ & $3.26 \mathrm{c}$ & $4.86 \mathrm{a}$ & $3.8 \mathrm{~b}$ \\
\hline Z38 & $429.20 \mathrm{a}$ & $1.2 \mathrm{a}$ & $1.8 \mathrm{a}$ & $14.72 \mathrm{~b}$ & $3.76 \mathrm{a}$ & $3.93 \mathrm{a}$ & $3.8 \mathrm{~b}$ \\
\hline Z29 & $591.72 \mathrm{a}$ & $1.0 \mathrm{a}$ & $0.8 \mathrm{~b}$ & $16.88 \mathrm{a}$ & $3.75 \mathrm{a}$ & $4.50 \mathrm{a}$ & $4.0 \mathrm{~b}$ \\
\hline Z40 & $579.88 \mathrm{a}$ & $1.4 \mathrm{a}$ & $0.8 \mathrm{~b}$ & $15.14 \mathrm{a}$ & $3.65 \mathrm{~b}$ & $4.14 \mathrm{a}$ & $3.4 \mathrm{c}$ \\
\hline Z35 & $283.56 \mathrm{~b}$ & $1.2 \mathrm{a}$ & $0.8 \mathrm{~b}$ & $8.82 \mathrm{c}$ & $3.53 \mathrm{~b}$ & $2.50 \mathrm{~b}$ & $3.6 \mathrm{~b}$ \\
\hline Z39 & $628.20 \mathrm{a}$ & $1.2 \mathrm{a}$ & $2.6 \mathrm{a}$ & $13.44 \mathrm{~b}$ & $3.92 \mathrm{a}$ & $3.46 \mathrm{~b}$ & $3.2 \mathrm{c}$ \\
\hline Peneirão & $441.00 \mathrm{a}$ & $1.2 \mathrm{a}$ & $1.4 \mathrm{a}$ & $17.96 \mathrm{a}$ & $3.87 \mathrm{a}$ & $4.66 \mathrm{a}$ & $3.8 \mathrm{~b}$ \\
\hline Pirata & $457.10 \mathrm{a}$ & $1.4 \mathrm{a}$ & $0.6 \mathrm{~b}$ & $16.00 \mathrm{a}$ & $3.56 \mathrm{~b}$ & $4.46 \mathrm{a}$ & $3.4 \mathrm{c}$ \\
\hline Bamburral & $532.98 \mathrm{a}$ & $1.2 \mathrm{a}$ & $1.6 \mathrm{a}$ & $16.98 \mathrm{a}$ & $3.84 \mathrm{a}$ & $4.46 \mathrm{a}$ & $3.6 \mathrm{~b}$ \\
\hline L80 & $317.46 \mathrm{~b}$ & $1.0 \mathrm{a}$ & $1.2 \mathrm{a}$ & $9.40 \mathrm{c}$ & $3.71 \mathrm{a}$ & $2.52 \mathrm{~b}$ & $2.6 \mathrm{~d}$ \\
\hline $\mathrm{AP}$ & $314.71 \mathrm{~b}$ & $1.4 \mathrm{a}$ & $0.0 \mathrm{~b}$ & $8.68 \mathrm{c}$ & $2.70 \mathrm{~d}$ & $3.22 \mathrm{~b}$ & $3.2 \mathrm{c}$ \\
\hline Tardio V & $293.96 \mathrm{~b}$ & $1.2 \mathrm{a}$ & $1.0 \mathrm{~b}$ & $14.10 \mathrm{~b}$ & $3.41 \mathrm{~b}$ & $4.13 \mathrm{a}$ & $3.4 \mathrm{c}$ \\
\hline Beira Rio & $372.28 \mathrm{~b}$ & $1.6 \mathrm{a}$ & $0.0 \mathrm{~b}$ & $15.94 \mathrm{a}$ & $3.15 \mathrm{c}$ & $5.05 \mathrm{a}$ & $3.2 \mathrm{c}$ \\
\hline Precoce V & $340.98 \mathrm{~b}$ & $1.2 \mathrm{a}$ & $1.2 \mathrm{a}$ & $13.74 \mathrm{~b}$ & $3.43 \mathrm{~b}$ & $4.03 \mathrm{a}$ & $3.2 \mathrm{c}$ \\
\hline AT Bahia & $263.62 \mathrm{~b}$ & $1.2 \mathrm{a}$ & $1.0 \mathrm{~b}$ & $10.10 \mathrm{c}$ & $3.28 \mathrm{c}$ & $3.09 \mathrm{~b}$ & $3.2 \mathrm{c}$ \\
\hline Graudão & $264.53 \mathrm{~b}$ & $1.4 \mathrm{a}$ & $0.6 \mathrm{~b}$ & $8.38 \mathrm{c}$ & $3.47 \mathrm{~b}$ & $2.41 \mathrm{~b}$ & $3.6 \mathrm{~b}$ \\
\hline Imbigudinho & $296.30 \mathrm{~b}$ & $1.2 \mathrm{a}$ & $0.8 \mathrm{~b}$ & $9.02 \mathrm{c}$ & $3.22 \mathrm{c}$ & $2.78 \mathrm{~b}$ & $3.0 \mathrm{c}$ \\
\hline CHR & $174.12 b$ & $1.6 \mathrm{a}$ & $0.4 \mathrm{~b}$ & $5.14 \mathrm{c}$ & $3.26 \mathrm{c}$ & $1.58 \mathrm{~b}$ & $2.8 \mathrm{~d}$ \\
\hline 700 & $432.58 \mathrm{a}$ & $1.0 \mathrm{a}$ & $0.0 \mathrm{~b}$ & $15.42 \mathrm{a}$ & $3.17 \mathrm{c}$ & $4.89 \mathrm{a}$ & $3.2 \mathrm{c}$ \\
\hline Alicrim & $261.80 \mathrm{~b}$ & $1.4 \mathrm{a}$ & $0.2 \mathrm{~b}$ & $7.50 \mathrm{c}$ & $2.69 \mathrm{~d}$ & $2.81 \mathrm{~b}$ & $2.2 \mathrm{~d}$ \\
\hline Bicudo & $383.79 \mathrm{~b}$ & $1.0 \mathrm{a}$ & $1.2 \mathrm{a}$ & $16.90 \mathrm{a}$ & $3.84 \mathrm{a}$ & $4.52 \mathrm{a}$ & $3.0 \mathrm{c}$ \\
\hline B01 & $215.90 \mathrm{~b}$ & $1.0 \mathrm{a}$ & $0.0 \mathrm{~b}$ & $12.60 \mathrm{~b}$ & $2.57 \mathrm{~d}$ & $4.96 \mathrm{a}$ & $3.6 \mathrm{~b}$ \\
\hline Verdinho R & $260.70 \mathrm{~b}$ & $1.0 \mathrm{a}$ & $0.0 \mathrm{~b}$ & $12.40 \mathrm{~b}$ & $2.98 \mathrm{c}$ & $4.16 \mathrm{a}$ & $3.8 \mathrm{~b}$ \\
\hline $\mathrm{P} 2$ & $479.98 \mathrm{a}$ & $1.0 \mathrm{a}$ & $0.4 \mathrm{~b}$ & $17.38 \mathrm{a}$ & $4.24 \mathrm{a}$ & $4.08 \mathrm{a}$ & $3.8 \mathrm{~b}$ \\
\hline Cheique & $482.72 \mathrm{a}$ & $1.4 \mathrm{a}$ & $1.4 \mathrm{a}$ & $14.42 \mathrm{~b}$ & $3.87 \mathrm{a}$ & $3.85 \mathrm{a}$ & $3.2 \mathrm{c}$ \\
\hline A1 & $493.20 \mathrm{a}$ & $1.0 \mathrm{a}$ & $2.0 \mathrm{a}$ & $11.22 \mathrm{c}$ & $3.90 \mathrm{a}$ & $2.81 \mathrm{~b}$ & $3.4 \mathrm{c}$ \\
\hline 02 Emcapa & $419.60 \mathrm{a}$ & $1.2 \mathrm{a}$ & $0.8 \mathrm{~b}$ & $17.14 \mathrm{a}$ & $3.44 \mathrm{~b}$ & $4.97 \mathrm{a}$ & $3.8 \mathrm{~b}$ \\
\hline P1 & $295.46 \mathrm{~b}$ & $1.2 \mathrm{a}$ & $0.6 \mathrm{~b}$ & $12.74 \mathrm{~b}$ & $3.41 \mathrm{~b}$ & $3.73 \mathrm{a}$ & $3.4 \mathrm{c}$ \\
\hline
\end{tabular}

${ }^{1}$ LA: Leaf area $\left(\mathrm{cm}^{2}\right) ;{ }^{2} \mathrm{NOS}$ : number of orthotropic shoots; ${ }^{3} \mathrm{NPTB}$ : number of plagiotropic branches; ${ }^{4} \mathrm{PSH}$ : primary stem height (cm); ${ }^{5} \mathrm{PSBD}$ : primary stem base diameter $(\mathrm{cm}) ;{ }^{6} \mathrm{PSH} / \mathrm{PSBD}$ ratio; ${ }^{7}$ NIPS: number of internodes of the primary stem. ${ }^{*}$ Means followed by the same letter in a column belong to the same cluster based on the ScottKnott clustering test at $5 \%$ probability.

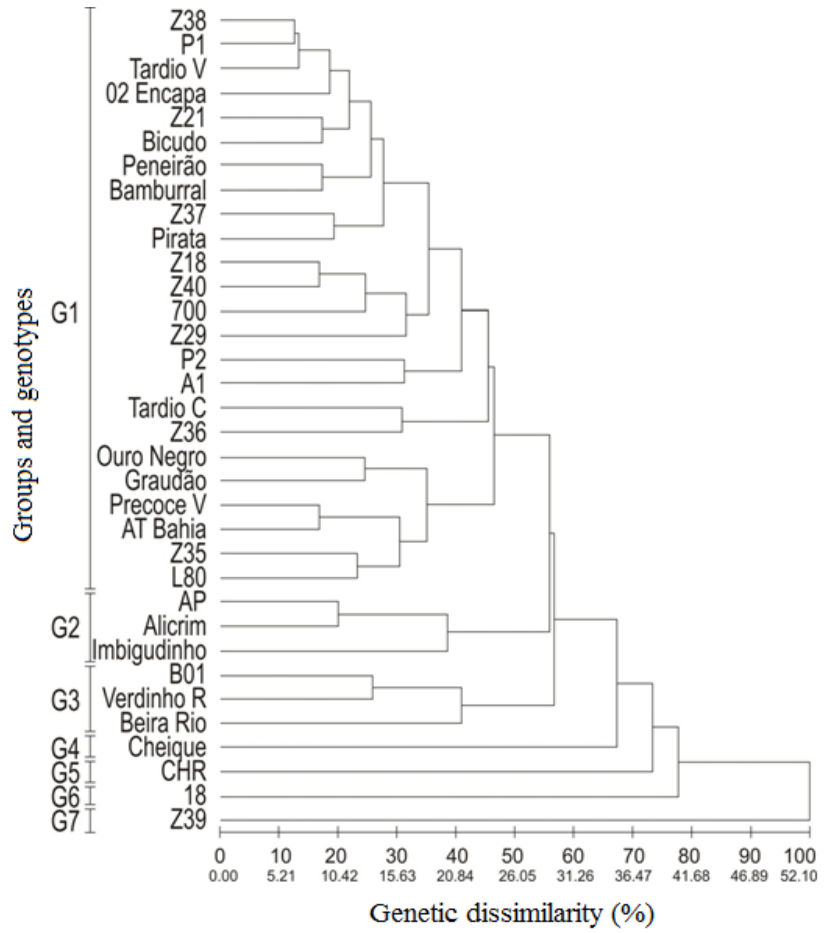

Fig 1. Dendrogram showing the genetic dissimilarity among the different genotypes of Conilon coffee, obtained through the unweighted pair group method with arithmetic mean (UPGMA), using the Mahalanobis distance $\left(\mathrm{D}^{2}\right)$. 
Table 2. Morphological characteristics of seedlings of different Conilon coffee genotypes

\begin{tabular}{|c|c|c|c|c|c|c|c|}
\hline Genotypes & $\mathrm{PSE}^{1}$ & $\mathrm{SSH}^{2}$ & $\mathrm{SSBD}^{3}$ & NISS $^{4}$ & $\mathrm{LLR}^{5}$ & $\mathrm{NPR}^{6}$ & $\mathrm{RV}^{7}$ \\
\hline Tardio C & $3.02 \mathrm{~b}^{*}$ & $3.94 \mathrm{a}$ & $2.33 \mathrm{a}$ & $1.6 \mathrm{a}$ & $15.5 \mathrm{~b}$ & $6.6 \mathrm{~b}$ & $3.0 \mathrm{~b}$ \\
\hline 18 & $3.08 \mathrm{~b}$ & $0.00 \mathrm{a}$ & $0.00 \mathrm{a}$ & $0.0 \mathrm{a}$ & $17.3 \mathrm{a}$ & $7.8 \mathrm{a}$ & $2.4 \mathrm{~b}$ \\
\hline Ouro Negro & $3.13 \mathrm{~b}$ & $1.80 \mathrm{a}$ & $0.61 \mathrm{a}$ & $0.4 \mathrm{a}$ & $19.2 \mathrm{a}$ & $7.8 \mathrm{a}$ & $3.8 \mathrm{~b}$ \\
\hline Z36 & $3.23 \mathrm{~b}$ & $0.46 \mathrm{a}$ & $0.53 \mathrm{a}$ & $0.4 \mathrm{a}$ & $16.5 \mathrm{a}$ & $7.0 \mathrm{a}$ & $2.4 \mathrm{~b}$ \\
\hline Z21 & $4.03 \mathrm{a}$ & $1.40 \mathrm{a}$ & $0.51 \mathrm{a}$ & $0.6 \mathrm{a}$ & $17.9 \mathrm{a}$ & $7.6 \mathrm{a}$ & $3.6 \mathrm{~b}$ \\
\hline Z37 & $4.51 \mathrm{a}$ & $3.18 \mathrm{a}$ & $1.89 \mathrm{a}$ & $1.6 \mathrm{a}$ & $17.6 \mathrm{a}$ & $7.8 \mathrm{a}$ & $2.6 \mathrm{~b}$ \\
\hline Z18 & $4.15 \mathrm{a}$ & $1.06 \mathrm{a}$ & $1.01 \mathrm{a}$ & $0.8 \mathrm{a}$ & $17.2 \mathrm{a}$ & $6.6 \mathrm{~b}$ & $3.2 \mathrm{~b}$ \\
\hline Z38 & $3.89 \mathrm{a}$ & $0.28 \mathrm{a}$ & $0.57 \mathrm{a}$ & $0.2 \mathrm{a}$ & $17.4 \mathrm{a}$ & $6.4 \mathrm{~b}$ & $3.2 \mathrm{~b}$ \\
\hline Z29 & $4.22 \mathrm{a}$ & $0.00 \mathrm{a}$ & $0.00 \mathrm{a}$ & $0.0 \mathrm{a}$ & $17.0 \mathrm{a}$ & $7.4 \mathrm{a}$ & $3.2 \mathrm{~b}$ \\
\hline Z40 & $4.40 \mathrm{a}$ & $0.60 \mathrm{a}$ & $0.97 \mathrm{a}$ & $0.6 \mathrm{a}$ & $16.0 \mathrm{a}$ & $6.0 \mathrm{~b}$ & $3.4 \mathrm{~b}$ \\
\hline Z35 & $2.49 \mathrm{~b}$ & $0.32 \mathrm{a}$ & $0.67 \mathrm{a}$ & $0.4 \mathrm{a}$ & $14.8 \mathrm{~b}$ & $5.0 \mathrm{~b}$ & $1.2 \mathrm{~b}$ \\
\hline Z39 & $4.25 \mathrm{a}$ & $1.16 \mathrm{a}$ & $0.65 \mathrm{a}$ & $0.4 \mathrm{a}$ & $14.8 \mathrm{~b}$ & $9.0 \mathrm{a}$ & $6.0 \mathrm{a}$ \\
\hline Peneirão & $4.76 \mathrm{a}$ & $0.26 \mathrm{a}$ & $0.53 \mathrm{a}$ & $0.2 \mathrm{a}$ & $13.2 \mathrm{~b}$ & $3.8 \mathrm{~b}$ & $3.2 \mathrm{~b}$ \\
\hline Pirata & $4.84 \mathrm{a}$ & $2.16 \mathrm{a}$ & $1.20 \mathrm{a}$ & $1.2 \mathrm{a}$ & $13.5 \mathrm{~b}$ & $5.4 \mathrm{~b}$ & $2.8 \mathrm{~b}$ \\
\hline Bamburral & $4.88 \mathrm{a}$ & $3.08 \mathrm{a}$ & $0.57 \mathrm{a}$ & $0.6 \mathrm{a}$ & $15.0 \mathrm{~b}$ & $5.0 \mathrm{~b}$ & $4.2 \mathrm{~b}$ \\
\hline L80 & $3.64 \mathrm{~b}$ & $0.00 \mathrm{a}$ & $0.00 \mathrm{a}$ & $0.0 \mathrm{a}$ & $13.2 \mathrm{~b}$ & $6.4 \mathrm{~b}$ & $2.4 \mathrm{~b}$ \\
\hline $\mathrm{AP}$ & $2.68 \mathrm{~b}$ & $0.56 \mathrm{a}$ & $0.93 \mathrm{a}$ & $0.6 \mathrm{a}$ & $16.6 \mathrm{a}$ & $7.8 \mathrm{a}$ & $2.0 \mathrm{~b}$ \\
\hline Tardio V & $4.13 \mathrm{a}$ & $0.32 \mathrm{a}$ & $0.54 \mathrm{a}$ & $0.2 \mathrm{a}$ & $15.8 \mathrm{a}$ & $6.4 \mathrm{~b}$ & $3.0 \mathrm{~b}$ \\
\hline Beira Rio & $4.99 \mathrm{a}$ & $1.30 \mathrm{a}$ & $1.24 \mathrm{a}$ & $0.8 \mathrm{a}$ & $13.8 \mathrm{~b}$ & $9.0 \mathrm{a}$ & $4.0 \mathrm{~b}$ \\
\hline Precoce V & $4.35 \mathrm{a}$ & $0.66 \mathrm{a}$ & $0.50 \mathrm{a}$ & $0.4 \mathrm{a}$ & $13.5 \mathrm{~b}$ & $7.8 \mathrm{a}$ & $3.8 \mathrm{~b}$ \\
\hline AT Bahia & $3.17 \mathrm{~b}$ & $0.22 \mathrm{a}$ & $0.59 \mathrm{a}$ & $0.2 \mathrm{a}$ & $15.8 \mathrm{a}$ & $6.4 \mathrm{~b}$ & $3.4 \mathrm{~b}$ \\
\hline Graudão & $2.35 \mathrm{~b}$ & $0.60 \mathrm{a}$ & $0.90 \mathrm{a}$ & $0.4 \mathrm{a}$ & $14.8 \mathrm{~b}$ & $7.4 \mathrm{a}$ & $2.8 \mathrm{~b}$ \\
\hline Imbigudinho & $4.23 \mathrm{a}$ & $0.28 \mathrm{a}$ & $0.46 \mathrm{a}$ & $0.2 \mathrm{a}$ & $14.2 \mathrm{~b}$ & $6.4 \mathrm{~b}$ & $3.0 \mathrm{~b}$ \\
\hline CHR & $1.90 \mathrm{~b}$ & $1.78 \mathrm{a}$ & $1.81 \mathrm{a}$ & $1.2 \mathrm{a}$ & $12.4 \mathrm{~b}$ & $5.2 \mathrm{~b}$ & $2.6 \mathrm{~b}$ \\
\hline 700 & $4.87 \mathrm{a}$ & $0.00 \mathrm{a}$ & $0.00 \mathrm{a}$ & $0.0 \mathrm{a}$ & $14.8 \mathrm{~b}$ & $6.4 \mathrm{~b}$ & $3.2 \mathrm{~b}$ \\
\hline Alicrim & $3.52 \mathrm{~b}$ & $2.00 \mathrm{a}$ & $1.00 \mathrm{a}$ & $1.0 \mathrm{a}$ & $15.0 \mathrm{~b}$ & $7.6 \mathrm{a}$ & $1.6 \mathrm{~b}$ \\
\hline Bicudo & $5.63 \mathrm{a}$ & $0.00 \mathrm{a}$ & $0.00 \mathrm{a}$ & $0.0 \mathrm{a}$ & $14.8 \mathrm{~b}$ & $7.8 \mathrm{a}$ & $2.2 \mathrm{~b}$ \\
\hline B01 & $3.48 \mathrm{~b}$ & $0.00 \mathrm{a}$ & $0.00 \mathrm{a}$ & $0.0 \mathrm{a}$ & $14.6 \mathrm{~b}$ & $7.0 \mathrm{a}$ & $2.8 \mathrm{~b}$ \\
\hline Verdinho R & $3.27 \mathrm{~b}$ & $0.00 \mathrm{a}$ & $0.00 \mathrm{a}$ & $0.0 \mathrm{a}$ & $14.1 \mathrm{~b}$ & $8.6 \mathrm{a}$ & $3.8 \mathrm{~b}$ \\
\hline $\mathrm{P} 2$ & $4.56 \mathrm{a}$ & $0.00 \mathrm{a}$ & $0.00 \mathrm{a}$ & $0.0 \mathrm{a}$ & $14.2 \mathrm{~b}$ & $9.6 \mathrm{a}$ & $6.4 \mathrm{a}$ \\
\hline Cheique & $4.57 \mathrm{a}$ & $1.58 \mathrm{a}$ & $0.60 \mathrm{a}$ & $1.3 \mathrm{a}$ & $12.1 \mathrm{~b}$ & $8.0 \mathrm{a}$ & $5.8 \mathrm{a}$ \\
\hline A1 & $3.27 \mathrm{~b}$ & $0.00 \mathrm{a}$ & $0.00 \mathrm{a}$ & $0.0 \mathrm{a}$ & $13.6 \mathrm{~b}$ & $7.8 \mathrm{a}$ & $6.0 \mathrm{a}$ \\
\hline 02 Emcapa & $4.49 \mathrm{a}$ & $1.52 \mathrm{a}$ & $0.60 \mathrm{a}$ & $0.6 \mathrm{a}$ & $19.8 \mathrm{a}$ & $7.6 \mathrm{a}$ & $4.0 \mathrm{~b}$ \\
\hline P1 & $3.71 \mathrm{~b}$ & $0.64 \mathrm{a}$ & $0.40 \mathrm{a}$ & $0.5 \mathrm{a}$ & $15.2 \mathrm{~b}$ & $4.6 \mathrm{~b}$ & $2.4 \mathrm{~b}$ \\
\hline
\end{tabular}

${ }^{1}$ PSE: primary stem etiolation (cm internodes $\left.{ }^{-1}\right) ;{ }^{2} \mathrm{SSH}$ : secondary stem height $(\mathrm{cm}) ;{ }^{3} \mathrm{SSBD}$ : secondary stem base diameter (mm); ${ }^{4} \mathrm{NISS}$ : number of internodes on secondary stem; ${ }^{5}$ LLR: length of largest root $(\mathrm{cm}):{ }^{6} \mathrm{NPR}$ : number of primary roots; ${ }^{7} \mathrm{RV}$ : root volume $\left(\mathrm{cm}^{3}\right)$. ${ }^{*}$ Means followed by the same letter in a column belong to the same cluster based on the Scott-Knott clustering test at $5 \%$ probability.

et al. (1960), the higher the value the DQI, the better the quality of the seedlings. In this case, quality indicates a better balance between the shoot and root systems, resulting in more seedlings adapted to the harsh conditions of the field, which ensures lower rates of death after planting (Andrade Júnior et al., 2013). In the production of Conilon coffee seedlings, this information can be useful in planning management strategies, given the differences in growth patterns of the 34 genotypes studied (Tables 1 and 2).

\section{Genetic divergence}

The characteristic LA exhibited significant phenotypic correlation with the majority of characteristics assessed, such as NPTB, PSH, PSBD, PSE, RV, LDM, SLM, SDM, ADM, RDM, TDM, ADM/RDM, and DQI (Table 4), indicating a strong influence over the characteristics linked to the biomass production of the plant. NOS was significantly correlated with SSH, SSBD and NISS, with $\mathrm{R}^{2}$ values of $0.75,0.97$ and 0.91 , respectively. NPTB exhibited a significant positive correlation with PSBD, RV, LDM, SDM, ADM, RDM, TDM, and DQI (Table 4). According to Carvalho et al. (2010), the number of plagiotropic branches experiences strong environmental effects because of the interaction between genotype and environment. The higher negative correlation (0.37) was between NIPS and NPR (Table 4). This result could be explained by the immaturity of the plants at the time of assessment, as 120 DAPC may have been insufficient for a positive correlation to be observed. The obtained highest magnitude (0.99) was observed for the correlations between ADM and LDM, and ADM and TDM. The DQI was significantly correlated with LA, NPTB, PSBD, RV, LDM, SDM, ADM, RDM, and TDM, indicating that a desirable seedling of Conilon coffee should exhibit high values for these characteristics (Table 4). Dardengo et al. (2013) also observed a strong correlation of the total dry matter and stem base diameter with DQI in Conilon coffee seedlings. Grouping by Tocher's optimisation method, based on the Mahalanobis distance using 22 characteristics, clustered the 34 genotypes into eight groups (Table 5). The number of groups demonstrates the broad variability among the genotypes tested. Fonseca et al. (2006), working with 32 genotypes of Conilon coffee, and Ivoglo et al. (2008), with 21 offspring that were genetically half Conilon coffee, observed the formation of three and four groups, respectively. Dias et al. (2005) evaluated 25 progenies of Arabica coffee, observed the formation of five distinct groups. Guedes et al. (2013), studying 12 samples of Arabica coffee of the Maragogipe variety, observed the formation of four distinct groups. Silva et al. (2015) evaluated 71 genotypes of Conilon coffee and 56 of Robusta coffee; they observed the formation of 13 and five distinct groups, respectively. The first group included 25 genotypes, which exhibited greater similarity under Tocher's optimisation method, among the 34 genotypes evaluated (Table 5). The remaining genotypes were divided into groups comprising only one or two genotypes. The genotypes Beira 
Table 3. Dry matter and Dickson quality index of seedlings of different genotypes of Conilon coffee.

\begin{tabular}{|c|c|c|c|c|c|c|c|c|}
\hline Genotypes & DLM $^{1}$ & SLM $^{2}$ & $\mathrm{SDM}^{3}$ & $\mathrm{ADM}^{4}$ & $\mathrm{RDM}^{5}$ & $\mathrm{TDM}^{6}$ & $\mathrm{ADM} / \mathrm{RDM}^{7}$ & $\mathrm{DQI}^{8}$ \\
\hline Tardio C & $1.82 \mathrm{a}^{*}$ & $3.45 \mathrm{a}$ & $0.45 \mathrm{a}$ & $2.27 \mathrm{a}$ & $0.53 \mathrm{~b}$ & $2.80 \mathrm{a}$ & $4.48 \mathrm{a}$ & $0.42 \mathrm{a}$ \\
\hline 18 & $1.33 \mathrm{~b}$ & $2.67 \mathrm{~b}$ & $0.28 \mathrm{~b}$ & $1.61 \mathrm{c}$ & $0.40 \mathrm{~b}$ & $2.01 \mathrm{~b}$ & $4.08 \mathrm{a}$ & $0.23 \mathrm{~b}$ \\
\hline Ouro Negro & $1.49 \mathrm{~b}$ & $3.05 \mathrm{~b}$ & $0.38 \mathrm{a}$ & $1.87 \mathrm{~b}$ & $0.68 \mathrm{a}$ & $2.56 \mathrm{a}$ & $2.92 \mathrm{~b}$ & $0.44 \mathrm{a}$ \\
\hline $\mathrm{Z} 36$ & $1.54 \mathrm{~b}$ & $3.22 \mathrm{~b}$ & $0.33 \mathrm{~b}$ & $1.87 \mathrm{~b}$ & $0.41 \mathrm{~b}$ & $2.28 \mathrm{a}$ & $4.57 \mathrm{a}$ & $0.31 \mathrm{~b}$ \\
\hline $\mathrm{Z} 21$ & $1.28 \mathrm{~b}$ & $3.00 \mathrm{~b}$ & $0.44 \mathrm{a}$ & $1.72 \mathrm{~b}$ & $0.59 \mathrm{a}$ & $2.31 \mathrm{a}$ & $3.29 \mathrm{~b}$ & $0.33 \mathrm{~b}$ \\
\hline Z37 & $1.63 \mathrm{~b}$ & $3.32 \mathrm{a}$ & $0.48 \mathrm{a}$ & $2.12 \mathrm{~b}$ & $0.48 \mathrm{~b}$ & $2.60 \mathrm{a}$ & $4.48 \mathrm{a}$ & $0.29 \mathrm{~b}$ \\
\hline Z18 & $1.60 \mathrm{~b}$ & $3.28 \mathrm{a}$ & $0.45 \mathrm{a}$ & $2.05 \mathrm{~b}$ & $0.49 \mathrm{~b}$ & $2.54 \mathrm{a}$ & $4.53 \mathrm{a}$ & $0.29 \mathrm{~b}$ \\
\hline Z38 & $1.47 \mathrm{~b}$ & $3.36 \mathrm{a}$ & $0.42 \mathrm{a}$ & $1.90 \mathrm{~b}$ & $0.56 \mathrm{~b}$ & $2.46 \mathrm{a}$ & $3.50 \mathrm{a}$ & $0.34 \mathrm{~b}$ \\
\hline Z29 & $1.91 \mathrm{a}$ & $3.23 \mathrm{~b}$ & $0.53 \mathrm{a}$ & $2.44 \mathrm{a}$ & $0.73 \mathrm{a}$ & $3.17 \mathrm{a}$ & $3.51 \mathrm{a}$ & $0.40 \mathrm{a}$ \\
\hline Z40 & $1.92 \mathrm{a}$ & $3.28 \mathrm{a}$ & $0.50 \mathrm{a}$ & $2.42 \mathrm{a}$ & $0.52 \mathrm{~b}$ & $2.94 \mathrm{a}$ & $4.63 \mathrm{a}$ & $0.35 \mathrm{~b}$ \\
\hline Z35 & $0.80 \mathrm{c}$ & $2.84 \mathrm{~b}$ & $0.22 \mathrm{~b}$ & $1.02 \mathrm{c}$ & $0.29 \mathrm{~b}$ & $1.31 \mathrm{~b}$ & $3.58 \mathrm{a}$ & $0.22 \mathrm{~b}$ \\
\hline Z39 & $2.49 \mathrm{a}$ & $3.95 \mathrm{a}$ & $0.67 \mathrm{a}$ & $3.16 \mathrm{a}$ & $0.73 \mathrm{a}$ & $3.89 \mathrm{a}$ & $4.45 \mathrm{a}$ & $0.50 \mathrm{a}$ \\
\hline Peneirão & $1.61 \mathrm{~b}$ & $3.56 \mathrm{a}$ & $0.52 \mathrm{a}$ & $2.12 \mathrm{~b}$ & $0.59 \mathrm{a}$ & $2.71 \mathrm{a}$ & $3.78 \mathrm{a}$ & $0.33 \mathrm{~b}$ \\
\hline Pirata & $1.35 \mathrm{~b}$ & $2.98 \mathrm{~b}$ & $0.43 \mathrm{a}$ & $1.79 \mathrm{~b}$ & $0.47 \mathrm{~b}$ & $2.26 \mathrm{a}$ & $3.87 \mathrm{a}$ & $0.28 \mathrm{~b}$ \\
\hline Bamburral & $1.82 \mathrm{~b}$ & $3.47 \mathrm{a}$ & $0.52 \mathrm{a}$ & $2.34 \mathrm{a}$ & $0.62 \mathrm{a}$ & $2.96 \mathrm{a}$ & $3.87 \mathrm{a}$ & $0.36 \mathrm{~b}$ \\
\hline L80 & $0.96 \mathrm{c}$ & $3.00 \mathrm{~b}$ & $0.27 \mathrm{~b}$ & $1.23 \mathrm{c}$ & $0.43 \mathrm{~b}$ & $1.66 \mathrm{~b}$ & $2.91 \mathrm{~b}$ & $0.30 \mathrm{~b}$ \\
\hline $\mathrm{AP}$ & $0.91 \mathrm{c}$ & $2.96 \mathrm{~b}$ & $0.14 \mathrm{~b}$ & $1.05 \mathrm{c}$ & $0.29 \mathrm{~b}$ & $1.34 \mathrm{~b}$ & $3.96 \mathrm{a}$ & $0.19 b$ \\
\hline Tardio V & $1.14 \mathrm{c}$ & $3.81 \mathrm{a}$ & $0.34 \mathrm{~b}$ & $1.48 \mathrm{c}$ & $0.42 \mathrm{~b}$ & $1.90 \mathrm{~b}$ & $3.77 \mathrm{a}$ & $0.25 \mathrm{~b}$ \\
\hline Beira Rio & $1.30 \mathrm{~b}$ & $3.42 \mathrm{a}$ & $0.25 \mathrm{~b}$ & $1.55 \mathrm{c}$ & $0.53 \mathrm{~b}$ & $2.08 \mathrm{~b}$ & $3.28 \mathrm{~b}$ & $0.26 \mathrm{~b}$ \\
\hline Precoce V & $1.01 \mathrm{c}$ & $2.97 \mathrm{~b}$ & $0.25 \mathrm{~b}$ & $1.26 \mathrm{c}$ & $0.58 \mathrm{a}$ & $1.85 \mathrm{~b}$ & $2.21 \mathrm{c}$ & $0.30 \mathrm{~b}$ \\
\hline AT Bahia & $0.83 \mathrm{c}$ & $3.15 \mathrm{~b}$ & $0.23 \mathrm{~b}$ & $1.06 \mathrm{c}$ & $0.49 \mathrm{~b}$ & $1.55 \mathrm{~b}$ & $2.15 \mathrm{c}$ & $0.30 \mathrm{~b}$ \\
\hline Graudão & $0.81 \mathrm{c}$ & $2.97 \mathrm{~b}$ & $0.19 \mathrm{~b}$ & $0.99 \mathrm{c}$ & $0.44 \mathrm{~b}$ & $1.44 \mathrm{~b}$ & $2.36 \mathrm{c}$ & $0.31 \mathrm{~b}$ \\
\hline Imbigudinho & $1.01 \mathrm{c}$ & $3.35 \mathrm{a}$ & $0.20 \mathrm{~b}$ & $1.21 \mathrm{c}$ & $0.46 \mathrm{~b}$ & $1.67 \mathrm{~b}$ & $2.80 \mathrm{~b}$ & $0.31 \mathrm{~b}$ \\
\hline CHR & $0.50 \mathrm{c}$ & $2.87 \mathrm{~b}$ & $0.14 \mathrm{~b}$ & $0.64 \mathrm{c}$ & $0.34 \mathrm{~b}$ & $0.98 \mathrm{~b}$ & $1.96 \mathrm{c}$ & $0.29 \mathrm{~b}$ \\
\hline 700 & $1.58 \mathrm{~b}$ & $3.54 \mathrm{a}$ & $0.35 \mathrm{~b}$ & $1.93 \mathrm{~b}$ & $0.52 \mathrm{~b}$ & $2.44 \mathrm{a}$ & $3.99 \mathrm{a}$ & $0.29 \mathrm{~b}$ \\
\hline Alicrim & $0.85 \mathrm{c}$ & $3.17 \mathrm{~b}$ & $0.16 \mathrm{~b}$ & $1.01 \mathrm{c}$ & $0.31 \mathrm{~b}$ & $1.32 \mathrm{~b}$ & $3.66 \mathrm{a}$ & $0.26 \mathrm{~b}$ \\
\hline Bicudo & $1.15 \mathrm{c}$ & $3.01 \mathrm{~b}$ & $0.42 \mathrm{a}$ & $1.56 \mathrm{c}$ & $0.44 \mathrm{~b}$ & $2.00 \mathrm{~b}$ & $3.89 \mathrm{a}$ & $0.26 \mathrm{~b}$ \\
\hline B01 & $0.69 \mathrm{c}$ & $3.11 \mathrm{~b}$ & $0.17 \mathrm{~b}$ & $0.86 \mathrm{c}$ & $0.32 \mathrm{~b}$ & $1.18 \mathrm{~b}$ & $3.28 \mathrm{~b}$ & $0.16 b$ \\
\hline Verdinho R & $0.88 \mathrm{c}$ & $3.46 \mathrm{a}$ & $0.21 \mathrm{~b}$ & $1.09 \mathrm{c}$ & $0.51 \mathrm{~b}$ & $1.60 \mathrm{~b}$ & $2.18 \mathrm{c}$ & $0.26 \mathrm{~b}$ \\
\hline $\mathrm{P} 2$ & $1.91 \mathrm{a}$ & $3.93 \mathrm{a}$ & $0.60 \mathrm{a}$ & $2.51 \mathrm{a}$ & $0.92 \mathrm{a}$ & $3.42 \mathrm{a}$ & $2.89 \mathrm{~b}$ & $0.50 \mathrm{a}$ \\
\hline Cheique & $1.36 \mathrm{~b}$ & $2.90 \mathrm{~b}$ & $0.45 \mathrm{a}$ & $1.81 \mathrm{~b}$ & $0.67 \mathrm{a}$ & $2.48 \mathrm{a}$ & $2.98 \mathrm{~b}$ & $0.41 \mathrm{a}$ \\
\hline A1 & $1.81 \mathrm{a}$ & $3.66 \mathrm{a}$ & $0.50 \mathrm{a}$ & $2.32 \mathrm{a}$ & $0.84 \mathrm{a}$ & $3.15 \mathrm{a}$ & $2.74 \mathrm{~b}$ & $0.56 \mathrm{a}$ \\
\hline 02 Emcapa & $1.44 \mathrm{~b}$ & $3.44 \mathrm{a}$ & $0.41 \mathrm{a}$ & $1.85 \mathrm{~b}$ & $0.50 \mathrm{~b}$ & $2.35 \mathrm{a}$ & $3.93 \mathrm{a}$ & $0.27 \mathrm{~b}$ \\
\hline P1 & $0.92 \mathrm{c}$ & $3.13 \mathrm{~b}$ & $0.27 \mathrm{~b}$ & $1.19 \mathrm{c}$ & $0.30 \mathrm{~b}$ & $1.50 \mathrm{~b}$ & $3.89 \mathrm{a}$ & $0.15 \mathrm{~b}$ \\
\hline
\end{tabular}

${ }^{1} \mathrm{LDM}$ : leaf dry matter (mg); ${ }^{2} \mathrm{SLM}$ : specific leaf mass (mg); ${ }^{3} \mathrm{SDM}$ : stem dry matter (mg); ${ }^{4} \mathrm{ADM}$ : aboveground dry matter (mg): ${ }^{5} \mathrm{RDM}$ : root dry matter (mg); ${ }^{6} \mathrm{TDM}$ : total dry matter (mg); ${ }^{7} \mathrm{ADM} / \mathrm{RDM}$ ratio; ${ }^{8} \mathrm{DQI}$ : Dickson quality index. "Means followed by the same letter in a column belong to the same cluster based on the Scott-Knott clustering test at $5 \%$ probability.

Rio, CHR, B01, 18, and Z39 remained in isolated groups, these being considered the more dissimilar, by Tocher's optimisation method (Table 5). The results of the UPGMA method (Figure 1) were similar to those of Tocher's optimisation method, particularly when a lower limit of 50\% similarity among genotypes was imposed. Guedes et al. (2013) and Ivoglo et al. (2008) also observed similarities among results obtained through Tocher's optimisation and UPGMA, especially when the lower limits were set at 45 and $15 \%$ similarity, respectively. It is noteworthy that the distribution of individuals in the dendrogram does not follow a criterion for group formation, since the main aspect of this method consists of the branches that are obtained (Cruz et al., 2004). Individuals are grouped in pairs, using the arithmetic means of dissimilarity, wherein the dendrogram prioritizes the genotypes with high similarity (Guedes et al., 2013). The UPGMA method allowed the formation of seven groups among the 34 genotypes tested (Fig. 1). The first group comprised Z38, P1, Tardio V, 02 Emcapa, Z21, Bicudo, Peneirão, Bamburral, Z37, Pirata, Z18, Z40, 700, Z29, P2, A1, Tardio C, Z36, Ouro Negro, Graudão, Precoce V, AT Bahia, Z35, and L80 (Fig. 1). Genotypes P2, AP, B01, Verdinho R, and Beira Rio were placed in different groups by Tocher's optimisation and UPGMA, thus indicating divergence between the two methods. Likewise, Guedes et al. (2013) and Ivoglo et al. (2008) also observed the formation of distinct groups using the UPGMA method. The genotypes CHR, 18, and Z39 remained isolated, corroborating the results obtained by Tocher's method (Table 5). In addition to exhibiting greater genetic distance (Fig. 1), they also presented broad divergence from one another. In general, the genotypes that clustered into more distant groups, with greater dissimilarity from each other, can be considered promising for future improvement programs as well as for the selection of genetic material for the formation of new varieties.

\section{Materials and Methods}

\section{Production of seedlings}

Seedlings of 34 genotypes of Conilon coffee (Coffea canephora Pierre ex A. Froehner) were evaluated, following technical recommendations and produced in a greenhouse certified by the Brazilian Ministry of Agriculture in Vila Valério, Espírito Santo, Brazil, at 120 metres altitude, latitude $18^{\circ} 54^{\prime} 35.83$ "S and longitude $40^{\circ} 14^{\prime} 31.89^{\prime \prime} \mathrm{W}$. The climate is tropical, hot and humid in the summer, with a dry winter. The mean annual temperature is $23^{\circ} \mathrm{C}$, and the mean maximum and minimum temperatures are $29^{\circ} \mathrm{C}$ and $18^{\circ} \mathrm{C}$, respectively (National Water Agency, 2015). New orthotropic shoots were collected from 34 genotypes. Thirty-three of these were selected by coffee farmers in northern Espírito Santo and one in southern Bahia, being promising and unrecorded in varieties, and these were grown along with genotype 02 of the Emcapa 8111 variety (Bragança et al., 2001). The seedlings 
Table 4. Phenotypic correlation coefficients among the morphological characteristics of seedling of different genotypes of Conilon coffee.

\begin{tabular}{|c|c|c|c|c|c|c|c|c|c|c|c|}
\hline Characteristic & $\mathrm{LA}^{1}$ & $\mathrm{NOS}^{2}$ & $\mathrm{NPTB}^{3}$ & $\mathrm{PSH}^{4}$ & $\mathrm{PSBD}^{5}$ & PSH/PSBD ${ }^{6}$ & NIPS $^{7}$ & $\mathrm{PSE}^{8}$ & $\mathrm{SSH}^{9}$ & SSBD $^{10}$ & NISS $^{11}$ \\
\hline LA & 1 & 0.01 & $0.50 * *$ & $0.52 * *$ & $0.66^{* *}$ & 0.32 & 0.30 & $0.45 * *$ & 0.26 & 0.02 & 0.11 \\
\hline NOS & & 1 & 0.02 & -0.29 & -0.08 & -0.30 & -0.26 & -0.17 & $0.75^{* *}$ & $0.97 * *$ & $0.91 * *$ \\
\hline NPTB & & & 1 & -0.09 & $0.61 * *$ & -0.32 & -0.17 & 0.05 & 0.18 & 0.05 & 0.05 \\
\hline PSH & & & & 1 & $0.40^{*}$ & $0.93 * *$ & $0.70 * *$ & $0.70 * *$ & -0.05 & -0.29 & -0.17 \\
\hline PSBD & & & & & 1 & 0.05 & 0.24 & $0.33^{*}$ & 0.15 & -0.03 & 0.02 \\
\hline PSH/PSBD & & & & & & 1 & $0.67 * *$ & $0.64 * *$ & -0.12 & -0.32 & -0.20 \\
\hline NIPS & & & & & & & 1 & 0.02 & -0.11 & -0.22 & -0.22 \\
\hline PSE & & & & & & & & 1 & 0.04 & -0.21 & -0.05 \\
\hline $\mathrm{SSH}$ & & & & & & & & & 1 & $0.80 * *$ & $0.87 * *$ \\
\hline SSBD & & & & & & & & & & 1 & $0.90 * *$ \\
\hline Characteristic & $\mathrm{LLR}^{12}$ & $\mathrm{NPR}^{13}$ & $\mathrm{RV}^{14}$ & $\mathrm{LDM}^{15}$ & SLM $^{16}$ & $\mathrm{SDM}^{17}$ & $\mathrm{ADM}^{18}$ & $\mathrm{RDM}^{19}$ & $\mathrm{TDM}^{20}$ & $\mathrm{ADM} / \mathrm{RDM}^{21}$ & $\mathrm{DQI}^{22}$ \\
\hline LA & 0.28 & 0.13 & $0.50 * *$ & $0.95 * *$ & $0.35^{*}$ & $0.82 * *$ & $0.95 * *$ & $0.65^{* *}$ & $0.92 * *$ & $0.59 * *$ & $0.65^{* *}$ \\
\hline NOS & -0.11 & 0.03 & -0.18 & -0.02 & -0.09 & -0.15 & -0.06 & -0.24 & -0.10 & 0.19 & -0.02 \\
\hline NPTB & -0.08 & 0.08 & $0.36^{*}$ & $0.52 * *$ & 0.28 & $0.41^{*}$ & $0.51 * *$ & $0.38^{*}$ & $0.50 * *$ & 0.21 & $0.62 * *$ \\
\hline PSH & $0.46^{* *}$ & -0.24 & $0.36^{*}$ & $0.47 * *$ & 0.20 & $0.75 * *$ & $0.56^{* *}$ & $0.46^{* *}$ & $0.57 * *$ & 0.30 & 0.07 \\
\hline PSBD & 0.03 & -0.01 & $0.56 * *$ & $0.65 * *$ & 0.28 & $0.75 * *$ & $0.70 * *$ & $0.70 * *$ & $0.73 * *$ & $0.12 * *$ & $0.72 * *$ \\
\hline PSH/PSBD & $0.47 * *$ & -0.23 & 0.19 & 0.27 & 0.11 & $0.51 * *$ & $0.34 *$ & 0.24 & $0.34^{*}$ & 0.29 & -0.19 \\
\hline NIPS & $0.67 * *$ & -0.37 & 0.12 & 0.20 & -0.11 & $0.49 * *$ & 0.29 & 0.27 & 0.30 & 0.09 & -0.02 \\
\hline PSE & -0.02 & 0.05 & $0.38^{*}$ & $0.47 * *$ & $0.37 *$ & $0.53 * *$ & $0.50 * *$ & $0.374 *$ & $0.49 * *$ & $0.33^{*}$ & 0.12 \\
\hline SSH & 0.05 & 0.03 & -0.01 & 0.22 & 0.01 & 0.13 & 0.20 & -0.01 & 0.17 & 0.28 & 0.16 \\
\hline SSBD & -0.05 & -0.02 & -0.23 & 0.01 & -0.06 & -0.11 & -0.02 & -0.25 & -0.07 & 0.22 & -0.01 \\
\hline NISS & -0.09 & 0.03 & -0.12 & 0.04 & -0.15 & -0.02 & 0.03 & -0.18 & -0.02 & 0.26 & 0.01 \\
\hline LLR & 1 & -0.17 & -0.13 & 0.19 & -0.08 & 0.32 & 0.23 & 0.03 & 0.20 & 0.36 & -0.13 \\
\hline NPR & & 1 & 0.36 & 0.19 & 0.23 & -0.16 & 0.10 & 0.26 & 0.14 & -0.09 & 0.32 \\
\hline RV & & & 1 & $0.60 * *$ & $0.55 * *$ & 0.62 & 0.62 & 0.88 & 0.71 & -0.18 & $0.78 * *$ \\
\hline LDM & & & & 1 & $0.61 * *$ & $0.83 * *$ & $0.99 * *$ & $0.72 * *$ & $0.97 * *$ & $0.55 * *$ & $0.72 * *$ \\
\hline SLM & & & & & 1 & $0.46^{* *}$ & $0.59 * *$ & $0.53 * *$ & $0.60 * *$ & 0.20 & 0.50 \\
\hline SDM & & & & & & 1 & $0.90 * *$ & $0.74 * *$ & $0.91 * *$ & $0.39 *$ & $0.60 * *$ \\
\hline $\mathrm{ADM}$ & & & & & & & 1 & $0.75 * *$ & $0.99 * *$ & $0.53 * *$ & $0.72 * *$ \\
\hline RDM & & & & & & & & 1 & $0.84 * *$ & -0.14 & $0.87 * *$ \\
\hline TDM & & & & & & & & & 1 & $0.41 *$ & $0.78^{* *}$ \\
\hline $\mathrm{ADM} / \mathrm{RDM}$ & & & & & & & & & & 1 & -0.07 \\
\hline DQI & & & & & & & & & & & 1 \\
\hline
\end{tabular}

Table 5. Representation of groups created by Tocher's optimisation method based on the Mahalanobis distance $\left(\mathrm{D}^{2}\right)$ among different Conilon coffee genotypes.

\begin{tabular}{ll}
\hline Groups & Genotypes \\
\hline 1 & Z38; P1; Tardio V; 2 Emcapa; Z21; Bicudo; Bamburral; Peneirão; Pirata; AT Bahia; A1; Z40; 700; Z18; Z37; \\
2 & Z29; Z35; L80; Tardio C; Z36; Precoce V; Ouro Negro; Verdinho R; Graudão; and AP \\
3 & Imbigudinho and Alicrim \\
4 & P2 and Cheique \\
5 & Beira Rio \\
6 & CHR \\
7 & B01 \\
8 & 18 \\
\hline
\end{tabular}

were assessed when they exhibited four or five pairs of leaves, at 120 days after planting by cutting (DAPC) in a greenhouse. The experimental design was completely randomised with five repetitions. Each treatment included one genotype, and each plot included one seedling.

\section{Evaluated Characteristics}

This study assessed the leaf area (LA), following Partelli et al. (2006); the number of orthotropic shoots (NOS) and the number of plagiotropic branches (NPTB) of the seedlings; the primary stem height (PSH); the secondary stem height ( $\mathrm{SSH}$ ), determined as the distance from the insertion point of the stem of the cutting to the apex; the primary stem base diameter (PSBD); the secondary stem base diameter (SSBD), measured as the second internode of each orthotropic shoot, from bottom to top; the relationship between the primary stem height and the primary stem base diameter (PSH/PSBD); the number of internodes on the primary stem (NIPS) and on the secondary stem (NISS); the etiolation of the main stem (EMS), derived from the ratio between PSH and NIPS; the length of largest root (LLR); the number of 
primary roots (NPR); and the root volume (RV), measured in a graduated cylinder. The seedlings were dried in a convection oven at $60^{\circ} \mathrm{C}$ until they reached a constant weight. They were then weighed on a precision balance to obtain the leaf dry matter (LDM), stem dry matter (SDM), aboveground dry matter (ADM), root dry matter (RDM), total dry matter (TDM) and the ratio of aboveground to root dry matter (ADM/RDM) (determined as the ratio between SDM and $\mathrm{RDM}$ ); specific leaf mass (SLM) (determined as the ratio between LDM and LA), and the Dickson quality index (DQI) using the equation: $\mathrm{DQI}=\mathrm{TDM} /[(\mathrm{PSH} / \mathrm{PSD})+$ (PSBD/RDM)] (Dickson et al., 1960).

\section{Statistical analysis}

Analysis of variance was performed individually for each characteristic to determine the existence of genetic variation among genotypes. The Scott-Knott test (5\%) was used to compare means. Phenotypic correlation was estimated for the assessed characteristics. Genetic diversity among offspring was determined using Tocher's optimisation method and the hierarchical unweighted pair group method with arithmetic mean (UPGMA). The array of genetic dissimilarity was found based on the Mahalanobis distance $\left(\mathrm{D}^{2}\right)$, which served as the foundation for the formation of the groups. All analyses were performed using the Genes software (Cruz, 2013).

\section{Conclusion}

The genotypes A1, P2, Z39, Ouro Negro, Tardio C, Cheique, and Z29 exhibited a higher Dickson quality index. There is genetic variability among the seedlings of the 34 Conilon coffee genotypes tested. The most dissimilar genotypes are $\mathrm{CHR}, 18$, and $\mathrm{Z39}$, which were isolated groups in all clustering methods.

\section{References}

ANA, Agência Nacional de Águas (2015) A bacia do Rio Doce: características da bacia. Available in: <http://www.ana.gov.br/cbhriodoce/bacia/caracterizacao.as p\#clima>. Accessed in: May 152015.

Andrade Júnior S, Alexandre RS, Schmildt ER, Partelli FL, Ferrão MAG, Mauri AL (2013) Comparison between grafting and cutting as vegetative propagation methods for conilon coffee plants. Acta Scient. 35:461-469.

Barbosa DHGS, Rodrigues WP, Vieira HD, Partelli FL, Viana AP (2014) Adaptability and stability of conilon coffee in areas of high altitude. Genet Mol Res. 13:78797888.

Bonomo P, Cruz CD, Viana JMS, Pereira AA, Oliveira AR, Carneiro PCS (2004) Avaliação de progênies obtidas de cruzamentos de descendentes do Híbrido de Timor com as cultivares Catuaí Vermelho e Catuaí Amarelo. Bragantia. 63:207-219.

Bragança SM, Carvalho CHS, Fonseca AFA, Ferrão RG (2001) 'Emcapa 8111', 'Emcapa 8121' e 'Emcapa 8131': variedades clonais de café conilon lançadas para o estado do Espírito Santo. Pesq Agropec Bras. 36:765-770.

Bustamante J, Casanova A, Romàn N, Monterrey C (2004) Estimación temprana del potencial de rendimiento en café (Coffea arabica L.) var. Bramón I. Bioagro. 16:3-8.

Carvalho AM, Mendes ANG, Carvalho GR, Botelho CE, Gonçalves FMA, Ferreira AD (2010) Correlação entre crescimento e produtividade de cultivares de café em diferentes regiões de Minas Gerais, Brasil. Pesq Agropec
Bras. 45:269-275.

Cecon PR, Silva FF, Ferreira A, Ferrão RG, Carneiro APS, Detmann E, Faria PN, Morais TSS (2008) Análise de medidas repetidas na avaliação de clones de café 'Conilon'. Pesq Agropec Bras. 43:1171-1176.

CONAB, Companhia Nacional de Abastecimento. Acompanhamento da Safra Brasileira de Café (2015) Safra 2015 Segundo Levantamento. Available in: <http://www.conab.gov.br/OlalaCMS/uploads/arquivos/ 15_06_10_09_24_57_boletim_cafe_junho_2015.pdf >. Accessed in: May 152015.

Conagin CHTM, Mendes AJT (1961) Pesquisas citológicas e genéticas em três espécies de Coffea; autoincompatibilidade em Coffea canephora. Bragantia. 20:787-804.

Contarato CC, Sobreira FM, Tomaz MA, Jesus Junior WC, Fonseca AFA, Ferrão MAG, Ferrão RG (2010) Evalution of the initial development of conilon coffee clones (Coffea canephora). Sci Agr. 11:65-71.

Covre AM, Partelli FL, Mauri AL, Dias MA (2013) Crescimento e desenvolvimento inicial de genótipos de café Conilon.Agro@mbiente.7:193-202.

Cruz CD (2013) Genes: a software package for analysis in experimental statistics and quantitative genetics. Acta Sci Agron. 35:271-276.

Cruz CD, Carvalho SP, Vencovsky R. Estudo sobre divergência genética. I. Fatores que afetam a predição do comportamento de híbridos. Rev Ceres. 41:178-182.

DaMatta FM, Ronchi CP, Maestri M, Barros RS (2007) Ecophysiology of coffee growth and production. Braz J Plant Physiol. 19:485-510.

Dardengo MCJD, Sousa EF, Reis EF, Gravina GA (2013) Crescimento e qualidade de mudas de café Conilon produzidas em diferentes recipientes e níveis de sombreamento. Coffee Sci. 8:500-509.

Davis AP, Tosh J, Ruch N, Fay MF (2011) Growing coffee: Psilanthus (Rubiaceae) subsumed on the basis of molecular and morphological data implications for the size, morphology, distribution and evolutionary history of Coffea. Bot J Linn Soc. 167:357-377.

Denoeud F, Carretero-Paulet L, Dereeper A, Droc G, Guyot R, Pietrella M, Zheng C, Alberti A, Anthony F, Aprea G, Aury JM, Bento P, Bernard M, Bocs S, Campa C, Cenci A, Combes MC, Crouzillat D, Silva C, Daddiego L, Bellis F, Dussert S, Garsmeur O, Gayraud T, Guignon V, Jahn K, Jamilloux $\mathrm{V}$, Joët $\mathrm{T}$, Labadie $\mathrm{K}$, Lan $\mathrm{T}$, Leclercq $\mathrm{J}$, Lepelley M, Leroy T, Li LT, Librado P, Lopez L, Muñoz A, Noel B, Pallavicini A, Perrotta G, Poncet V, Pot D, Priyono, Rigoreau M, Rouard M, Rozas J, TranchantDubreuil C, Vanburen R, Zhang Q, Andrade AC, Argout X, Bertrand B, Kochko A, Graziosi G, Henry RJ, Jayarama, Ming R, Nagai C, Rounsley S, Sankoff D, Giuliano G, Albert VA, Wincker P, Lashermes P (2014) The coffee genome provides insight into the convergent evolution of caffeine biosynthesis. Science. 345:1181-1184.

Dias FP, Carvalho SP, Mendes ANG, Souza CAS, Raso BSM, Botelho CE. Caracterização de progênies do cafeeiro (Coffea arabica L.) selecionadas em Minas Gerais: III Divergência genética. Rev Ceres. 52:101-114.

Dickson A, Leaf AL, Hosner JF (1960) Quality appraisal of white spruce and white pine seedling stock in nurseries. Forest Chron. 36:10-13.

Fonseca AFA, Ferrão MAG, Ferrão RG, Verdin Filho AC, Volpi OS, Zucateli F (2004) 'Conilon Vitória - Incaper 8142': improved Coffea canephora var. Kouillou clone cultivar for the state of Espírito Santo. Crop Breed Appl Biotechnol. 4:503-505. 
Fonseca AFA, Sediyama T, Cruz CD, Sakaiyama NS, Ferrão MAG, Ferrão RG, Bragança SM (2006) Divergência genética em café conilon. Pesq Agropec Bras. 41:599-605.

Guedes JM, Vilela DJM, Rezende JC, Silva FL, Botelho CE, Carvalho SP (2013) Divergência genética entre cafeeiros do germoplasma Maragogipe. Bragantia. 72:127-132.

ICO, International Coffee Organization (2015) Trade statistics. Available in: http://www.ico.org/trade_ statistics.asp. Accessed in: May 152015.

Ivoglo MG, Fazuoli LC, Oliveira ACB, Gallo PB, Mistro JC, Silvarolla MB, Toma-Braghini M (2008) Divergência genética entre progênies de café Conilon. Bragantia. 67:823-831.

Oliveira ACB, Pereira AA, Silva FL, Rezende JC (2011) Prediction of genetic gains from selection in Arabica coffee progenies. Crop Breed Appl Biotechnol. 11:106-113.

Paiva AV, Poggiani F, Gonçalves JLM, Ferraz AV (2009) Crescimento de mudas de espécies arbóreas nativas, adubadas com diferentes doses de lodo de esgoto seco e com fertilização mineral. Sci Forestalis. 37:499-511.

Paiva RF, Mendes ANG, Carvalho GR, Rezende JC, Ferreira AD, Carvalho AM (2012) Comportamento de cultivares de cafeeiros $C$. arabica L. enxertados sobre cultivar 'Apoatã IAC 2258' (Coffea canephora). Ci Rural. 42:155-1160.

Partelli FL, Covre AM, Oliveira MG, Alexandre RS, Vitoria EL, Silva MB (2014a) Root system distribution and yield of 'Conilon' coffee propagated by seeds or cuttings. Pesq Agropec Bras. 49:349-355.
Partelli FL, Espindula MC, Marré WB, Vieira HD (2014b) Dry matter and macronutrient accumulation in fruits of Conilon coffee with different ripening cycles. Rev Bras Ciênc Solo. 38:214-222.

Partelli FL, Vieira HD, Detmann E, Campostrini E (2006) Estimativa da área foliar do cafeeiro a partir do comprimento da folha. Rev Ceres. 53:204-210.

Rodrigues WP, Vieira HD, Barbosa DHSG, Sousa Filho GR, Partelli FL (2014) Agronomic performance of arabica coffee genotypes in northwest Rio de Janeiro State. Genet Mol Res. 13:5664-5673.

Schimidt R, Dias JRM, Espíndula MC, Partelli FL, Alves ER (2015) Poda apical e vergamento da haste principal na formação de cafeeiros clonais. Coffee Sci. 10: 266-270.

Silva FL, Baffa DCF, Rezende JC, Oliveira ACB, Pereira AA, Cruz CD. Variabilidade genética entre genótipos de café robusta no estado de Minas Gerais. Coffee Sci. 10:2027.

Tomaz MA, Sakiyama NS, Martinez HEP, Cruz CD, Pereira AA, Freitas RS (2005) Porta-enxerto afetando o desenvolvimento de plantas de Coffea arabica L. Ci Rural. 35:570-575.

Weigel D, Jurgens G (2002) Stem cells that make stems. Nature. 415:751-754. 